\title{
Clinico-epidemiological study of Schistosomiasis mansoni in Waja-Timuga, District of Alamata, northern Ethiopia
}

Nigus Abebe ${ }^{1}$, Berhanu Erko², Girmay Medhin ${ }^{2}$ and Nega Berhe ${ }^{2^{*}}$

\begin{abstract}
Background: Intestinal schistosomiasis, caused by digenetic trematodes of the genus Schistosoma, is the most prevalent water related disease that causes considerable morbidity and mortality. Although prevalence of Schistosoma mansoni infection has been reported for the present study area, earlier studies have not estimated intensity of infections in relation to periportal fibrosis, which would have been crucial for epidemiological and clinical evaluations. Hence, a community based cross sectional study was conducted from December 2011 to March 2012 to assess prevalence of infection and schistosomal periportal fibrosis in Waja-Timuga, northern Ethiopia.

Methods: In a cross sectional study involving 371 randomly selected individuals, fresh stool samples were collected and processed by the Kato-Katz method and examined microscopically. Ultrasonography was used to determine status of schistosomal periportal fibrosis and to detect hepatomegaly and/or splenomegaly. Serum was collected for assay of hepatic activity. Statistical analysis was performed using STATA 11 statistical soft ware. P-value $<0.05$ was reported as statistically significant.

Results: The prevalence of S.mansoni infection was $73.9 \%$, while the prevalence of schistosomal periportal fibrosis was $12.3 \%$ and mean intensity of infection was 234 eggs per gram of stool. Peak prevalence and intensity of $S$. mansoni infection was documented in the age range of 10-20 years. Among the study individuals, hepatomegaly was recorded in $3.7 \%$ and splenomegaly was recorded in $7.4 \%$ of the study individuals. Similarly, among the study individuals who had definite periportal fibrosis, 5.9\% had elevated liver enzyme levels.

Conclusion: The high prevalence of Schistosoma mansoni infection and schistosomal periportal fibrosis observed in the study area calls for a periodic deworming program to reduce disease, morbidity and transmission. Preventive chemotherapy complemented with other control measures is highly required for sustainable control of schistosomiasis in the study area.
\end{abstract}

Keywords: Schistosoma mansoni, Intensity, Periportal fibrosis, Waja-Timuga, Ethiopia

\section{Background}

Intestinal Schistosomiasis is caused by S. mansoni and transmission occurs when the human definitive host skin comes in contact with cercariae of Biomphalaria snails. The disease remains one of the highly neglected tropical diseases, which causes high morbidity and considerable mortality in sub-Saharan Africa [1,2].

\footnotetext{
* Correspondence: nega_berhe@yahoo.com

${ }^{2}$ Addis Ababa University, Aklilu Lemma Institute of Pathobiology, Addis

Ababa, Ethiopia

Full list of author information is available at the end of the article
}

In endemic areas, children carry the largest burden of the disease characteristics of acute schistosomiasis are rarely seen; however, the adult population carries the chronic form of the disease characterized by periportal fibrosis, hepatomegaly, splenomegaly, portal hypertension, hematemesis and oesopheageal varices $[3,4]$.

Morbidity and mortality due to schistosomiasis are largely due to the consequences of a host $\mathrm{T}$ cell-mediated immune response against parasite eggs trapped in the tissue. Antigens released from the egg stimulate a granulomatous reaction involving $\mathrm{T}$ cells, macrophages, and eosinophils that results in clinical disease. However, the

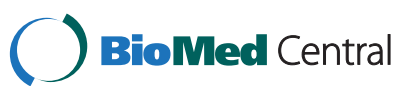


magnitude of the resulting granulomatous and fibrosing inflammation varies greatly from individual to individual [5], which seems to be influenced to a large extent by the nature of the induced immune response and its effects on granuloma formation and associated pathologies in target organs. In addition, the extent of morbidity differs markedly among individuals of similar ages with similar intensities of infection, both between and within communities: other factors may, therefore, also be involved, including host and parasite genetic differences, nutritional status, exposure to other infectious agents, and maternal infection status [6,7]. The degree of morbidity associated with S.mansoni is related to the intensity of infection and other factors. Symptoms and signs depend on the number and location of eggs trapped in the tissues. Initially, the inflammatory reaction is readily reversible. In the latter stages of the disease, the pathology is associated with collagen deposition and fibrosis, resulting in organ damage that may be only partially reversible. In light infection, individuals remain asymptomatic except in infants where bloody stools are seen, in mild infections, bloody stool, abdominal pain and nausea can occur, while in the heavy infections, individuals show lesions of the liver and spleen [8-10].

Ultrasonography is currently the diagnostic tool of choice for detecting pathologic conditions associated with schistosomiasis mansoni such as liver fibrosis and enlargement and dilatation of the portal vein $[11,12]$. Moreover, it provides sensitive and precise measurements of S.mansoni-associated pathologic changes [13].

In Ethiopia, the distribution of schistosomiasis is highly focal and varies from region to region because of several environmental, social and geographical factors [14]. The spread of schistosomiasis in Ethiopia is mainly attributed to water resource development and increasing population movement [15]. Although prevalence of schistosomiasis mansoni has been reported for the current study area [16], community level information on the epidemiology of the infection and schistosomal periportal fibrosis (PPF) is scarce for most endemic foci including the present study area. Therefore, the objective of the current study was to determine the prevalence of schistosomiasis mansoni and schistosomal PPF in Waja-Timuga, District of Alamata, northern Ethiopia.

\section{Methods}

\section{Study area and population}

The current study was conducted in southern Tigray, Alamata District, Waja-Timuga area, which is located $600 \mathrm{~km}$ north of Addis Ababa, the capital city of Ethiopia, and about $185 \mathrm{~km}$ south of Mekelle, the capital of the Tigray Regional State (Figure 1). Waja-Timuga has a total population of 11,030 (1051 households) with area coverage of $5985.5 \mathrm{~km}^{2}$ and about 10 tabias (local administrative units). The area is located between latitude of $12^{\circ} 19^{\prime} \mathrm{N}$ and longitude of $39036^{\prime} \mathrm{E}$ at $1470 \mathrm{~m}$ above sea level [17]. The inhabitants of the study area earn their living as government employees, farmers and self-employed (merchant, daily labourer etc.).

\section{Study design, sample size determination and sampling method}

This was a community based cross sectional study conducted between December 2011 - March 2012.

Sample size was determined based on the previous prevalence of $67.95 \%$ obtained from a study conducted among schoolchildren in Timuga and Waja, northern Ethiopia [18]. A 95\% level of confidence $(\mathrm{z}=1.96)$ and $5 \%$ margin of error (d) was used. With these assumptions the minimum sample size required for the study was 334 and with $11 \%$ allowed for non-respondents, the required sample size increased to 371 .

A list of 1051 households obtained from the local administrative units served as the sampling frame. A simple random sampling method was used to select study participating households. Using this method, one-hundred-fifty households from three accessible communities namely; 28 households from Waja, 71 households from Timuga and 51 households from Aroresha were selected. All family members were invited for S.mansoni infection examination.

\section{Stool collection and examination}

Fresh stool specimens were collected using pieces of plastic sheets distributed to each of the selected individuals. Specimens were processed for microscopic examination after $24 \mathrm{hrs}$ of smear preparation for evaluation of S. mansoni eggs by the quantitative Kato-Katz method using a template delivering $41.7 \mathrm{mg}$ of stool [19].

Two thick Kato-Katz smears were prepared from a single stool sample. The slides were labeled and transported using slide boxes to the parasitology laboratory at The College of Veterinary Medicine, Mekelle University for microscopic examination. Egg counts per slide were multiplied by 24 to convert into number of eggs per gram (EPG) of stool [19].

For quality control, $10 \%$ of the slides were randomly selected and re-examined by an experienced laboratory technician who was blinded to schistosome infection status of the individual. Any discrepancies were counter checked before results were recorded.

The intensity of infection was classified according to WHO [20] as light (1-99 EPG), moderate (100-400 EPG) and heavy infections (more than $400 \mathrm{EPG}$ ).

\section{Ultrasonography}

Ultrasonographic assessments were performed to detect pathological changes associated with S.mansoni infection in individuals who participated in parasitological 


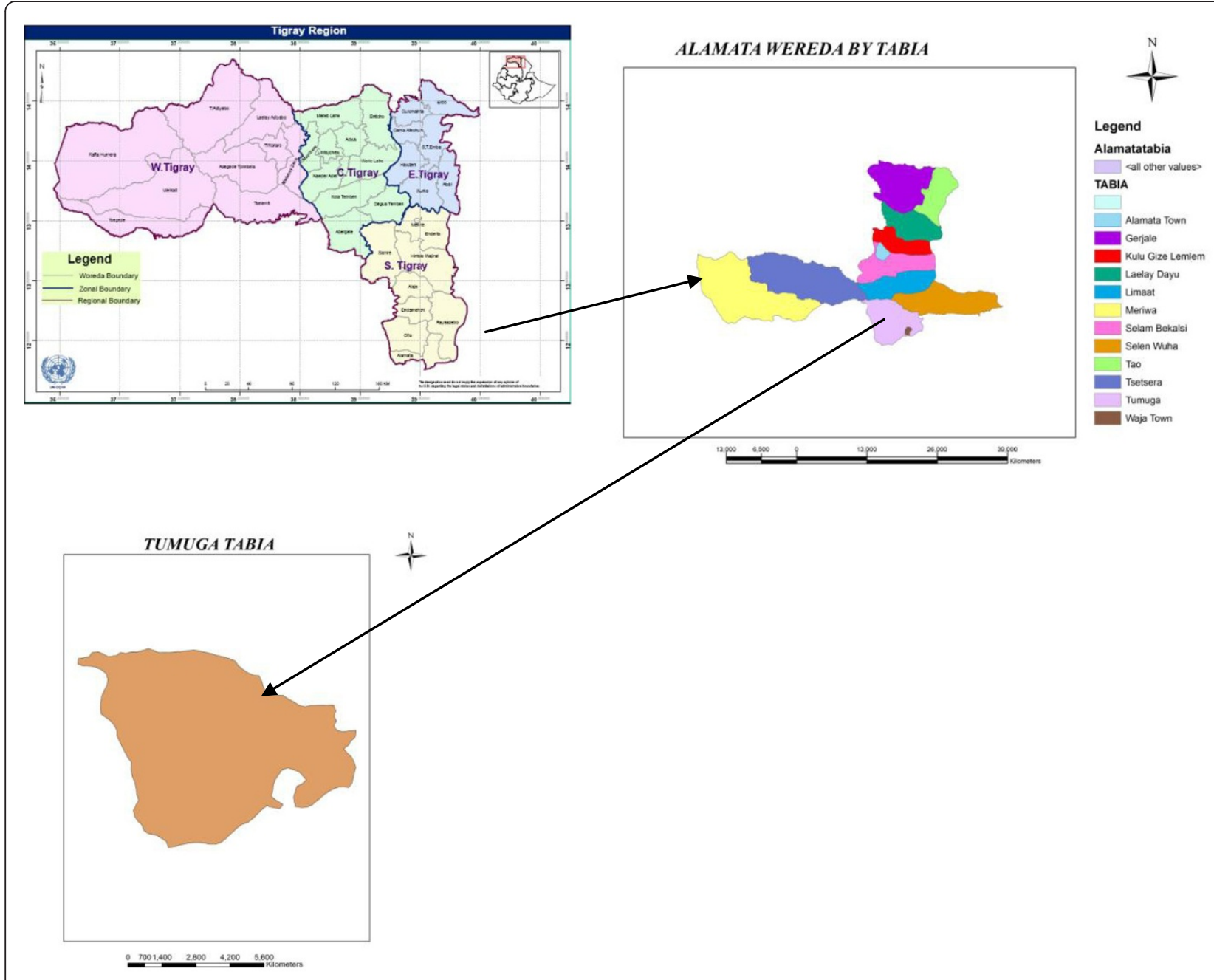

Figure 10 Map of study site.

screening. Using the WHO-Niamey protocol [21], standard ultrasonographic liver scans were performed using Hitachi EUB 405 portable ultrasound equipment (Tokyo, Japan), fitted with a $3.5-\mathrm{MHz}$ convex abdominal probe. All examinations were performed by the same clinician, who was blinded to the schistosome infections status of the individual.

In individuals with image patterns suggestive of PPF, the liver picture was compared with standard images and the corresponding image pattern score was recorded. In addition, assessment of the periportal thickening was made by taking inner to inner and outer to outer portal branch wall thickness (PBWT) measurements of branching portal veins. These measurements were close to branching points of the vessels, and the arithmetic mean differences between outer and inner wall thickness were taken as the individual's PBWT value. The summation of the image pattern and PBWT scores gave the final PPF grading of each individual. Accordingly, individuals with no/non-specific diffuse echogenic liver pattern associated with minimal wall thickening were classified as having "no PPF", those with image patterns suggestive of PPF but with wall thickness less or equal to mean \pm SD of normal PBWT-for height standard were classified "indeterminate PPF", and those with definite or advanced PPF by both image pattern and PBWT measurements were classified as "definite PPF" (Additional file 1).

\section{Serum collection and examination}

Five milliliters $(\mathrm{ml})$ of venous blood was collected from individuals whose ultrasound results were suggestive of image pattern PPF. Serum was separated by centrifugation and stored in thermo boxes at $-20^{\circ} \mathrm{C}$ in a freezer prior to dispatching to the Aklilu Lemma Institute of Pathobiology, Addis Ababa University. Levels of serum glutamic-oxaloacetic transaminase (SGOT) and serum glutamate-pyruvate transaminase (SGPT) was determined using a photometer (Photometer 5010, Germany) as 
biomarkers of liver function test. The ranges of SGOT and SGPT numbers may differ slightly depending on the technique and protocols used by different laboratories. Nevertheless, normal reference ranges were routinely provided by each laboratory and printed in the report. Although sources vary on specific reference range values for patients, $10-40 \mathrm{IU} / \mathrm{L}$ is the standard reference range for experimental studies [22].

\section{Ethical consideration}

The study was ethically approved by the Institutional Review Board of Aklilu Lemma Institute of Pathobiology, Addis Ababa University and a supportive letter was obtained from Tigray Regional State Health Bureau and Alamata District Health Office. Moreover, the aim of the study was explained to the study participants and written and verbal consent was obtained. Those individuals who were found positive for S.mansoni infection were treated with praziquantel $(40 \mathrm{mg} / \mathrm{kg}$ body weight) according to the WHO guideline without incurring any cost on the study individuals. Similarly those who were found positive for soil-transmitted helminths were treated with albendazole (single dose of $400 \mathrm{mg}$ ).

\section{Data analysis}

A data-base was developed to store quantitative data using Microsoft Office Excel 2007 spreadsheets and imported to statistical software. STATA version 11 was used to compute descriptive statistics of variables collected during the study. An independent t-test was carried out to compare the mean intensity of schistosomiasis among male and female study individuals. One way ANOVA was also used to compare the differences in intensity among age groups of the study individuals. P-value $<0.05$ was reported as statistically significant.

\section{Results}

Demographic characteristics of the study individuals

A total of 371 individuals ( male $=211$ and female $=160$ ) from three communities participated in this study. The majority of them (68.2\%) were residents of semi-urban (Waja and Timuga) settings and the remaining individuals were from Aroresha, which is a rural type of setting. The mean age of study individuals was $22.2(\mathrm{sd}=15.7)$ years.

\section{Schistosoma mansoni results}

The mean intensity of $S$. mansoni infection among study participants in Waja-Timuga was $234(\mathrm{sd}=431)$ EPG. There was a statistically significant difference $(\mathrm{p}<0.001)$ in intensity of S.mansoni infection between females $($ mean EPG $=174$, sd $=261)$ and males $($ mean $\mathrm{EPG}=279$, $\mathrm{sd}=521$ ) (Figure 2). However, there was no statistically significant difference in the mean EPG of rural and urban residents (140.0 versus 169.6).

A significant difference was observed between the intensity of S.mansoni infection between residents of Waja and Aroresha areas $\left(x^{2}=33.7, p<0.001\right)$. In the Waja area $27 \%, 19 \%$ and $19 \%$ of the study individuals had light, moderate and heavy infections, respectively. Among study individuals recruited from the Timuga area and of those infected, 29.4\% had light infection, 19\% had moderate infection and $19.6 \%$ had heavy infection. On the other hand, in the Aroresha area 32.2\%, 42.4\% and 14.4\% had light, moderate and heavy infections, respectively. Intensity of infection did not significantly differ between sexes $(P=0.218)$ (Figure 3$)$.

The highest intensity of S.mansoni infection was observed in the age range of 10.1-20.0 years followed by the age range of $1-10$ yeas (Table 1 ).

\section{Ultrasonography}

Of all the individuals $(\mathrm{n}=371)$ participating in parasitological screening, only 162 individuals were examined

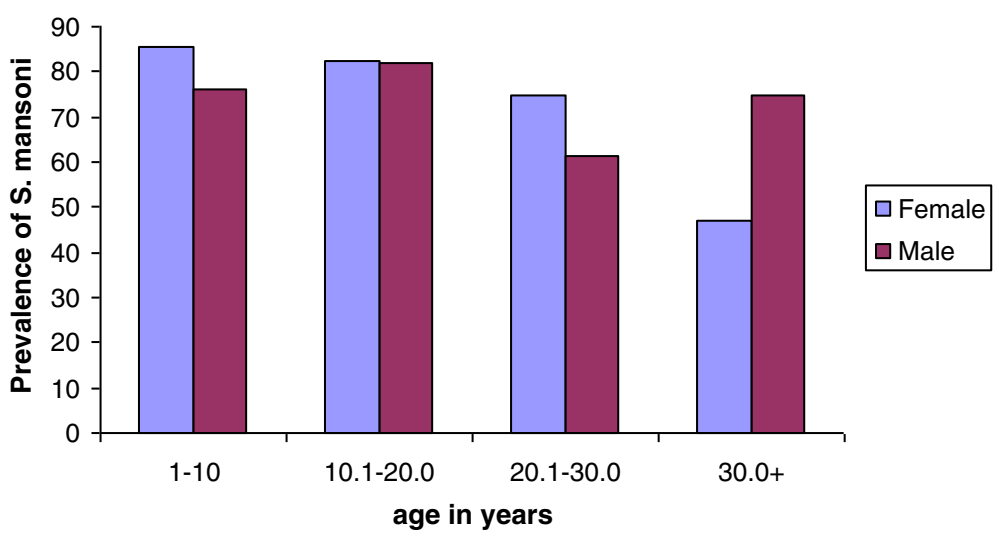

Figure 2 Prevalence of S. mansoni by sex, Waja-Timuga, Dec. 2011-Mar. 2012. 


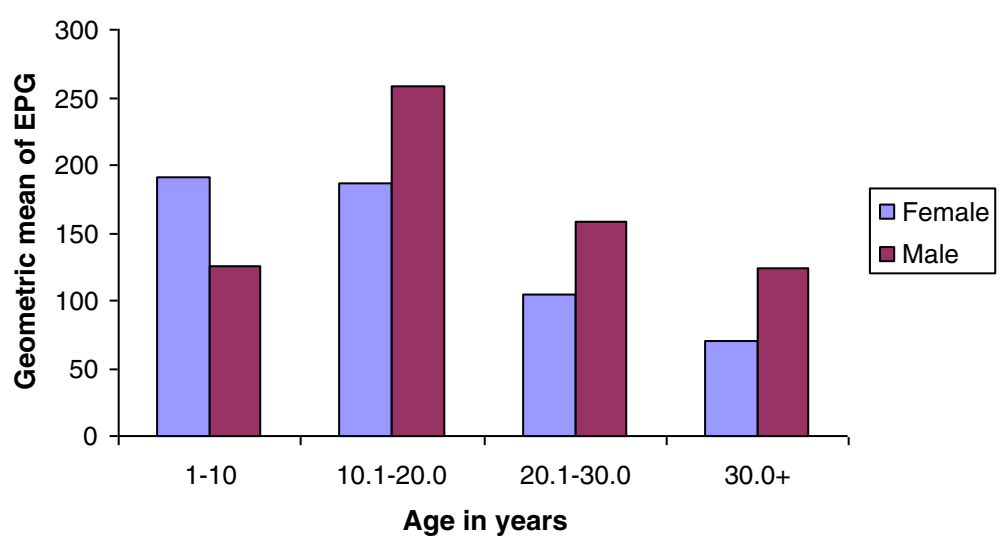

Figure 3 Geometric mean as a measure of intensity of infection of S. mansoni by sex, Waja-Timuga, Dec. 2011-Mar. 2012.

using ultrasonography for detection of schistosomal periportal fibrosis.

PPF status by parasitological characteristics is shown in Table 2. Final PPF status of study individuals was determined using a combination of results of image pattern ultrasound and PBWT. Based on this, over all prevalence of PPF status was $12.3 \%$. Presence/absence of ova of S.mansoni were not significantly associated with PPF status $(\mathrm{p}=0.238)$. Among egg positive individuals, 54.9\% had no PPF, $8.0 \%$ had indeterminate PPF and $11.1 \%$ had definite PFF.

Age and PPF status of the study individuals were significantly associated $(\mathrm{p}=0.001)$. Among study individuals who had S.mansoni infection, prevalence of PPF had a sharp rise in the age range of 10.1 to 20 years and reached its peak in the age range of 20.1 to 30 years (Figure 4a). Whereas, statistical significant association was not observed ( $\mathrm{p}=0.297$ ) between PPF status and intensity of infection (Figure 4b).

Prevalence of hepatomegaly and splenomegaly (results obtained from ultrasonography) stratified by PPF status is summarized in Figure 5. The overall prevalence of hepatomegaly was $3.7 \%$ and that of splenomegaly was $7.4 \%$. There was a demonstrable $S$. mansoni egg count in $83.3 \%$ and $91.7 \%$ of individuals who developed hepatomegaly and splenomegaly, respectively.

Table 1 Summary of age specific intensity of S.mansoni infection, Waja-Timuga, Dec.2011-Mar.2012

\begin{tabular}{lcccc}
\hline $\begin{array}{l}\text { Age category } \\
\text { years) }\end{array}$ & \multicolumn{2}{c}{ Level of Intensity of infection } & Mean \\
\cline { 2 - 4 } & Light & Moderate & Heavy & EPG \pm SD \\
\hline $1-10$ & $29.7 \%$ & $35.6 \%$ & $14.9 \%$ & $222.4 \pm 343.7$ \\
$10.1-20$ & $23.8 \%$ & $25.4 \%$ & $32.8 \%$ & $361.3 \pm 563.5$ \\
$20.1-30$ & $36.4 \%$ & $21.8 \%$ & $9.1 \%$ & $165.8 \pm 390.9$ \\
$>30$ & $33.3 \%$ & $20.4 \%$ & $6.5 \%$ & $119.5 \pm 273.3$ \\
\hline
\end{tabular}

\section{Liver enzyme level test}

Table 3 shows the proportion of study individuals with liver enzyme levels of SGOT and SGPT stratified by the categories of PPF status. Chemical analysis of SGOT and SGPT revealed that $8 \%(2 / 25)$ and $4 \%(1 / 25)$, respectively, were elevated from the normal ranges. Among the study individuals who had definite fibrosis $5.88 \%$ had elevated SGOT as well as SGPT. However, there was no statistically significant association between PPF status and liver enzyme level $(\mathrm{p}=0.49)$.

\section{Discussion}

In the current study, prevalence of schistosomiasis mansoni was $73.9 \%$ and mean intensity of infection was 234 eggs per gram of stool. Based on the available evidence, the prevalence of $S$. mansoni infection varied greatly from one region to another [23-25]. Occupational difference is often reflected in schistosomiasis prevalence as occupational groups performing intensive contact with cercariae-infested water bodies have higher rates of infection [26,27]. Most previous community studies showed that males were more affected by schistosomiasis than females as the result differential frequency of contact with water bodies $[28,29]$. However, in the current study there was no significant difference in the prevalence of S. mansoni infection between males and females, which is also

Table 2 Categories of PPF by parasitological characteristics of S.mansoni infection, Waja-Timuga, Dec. 2011- Mar. 2012

\begin{tabular}{lcccc}
\hline $\begin{array}{l}\text { S. mansoni } \\
\text { infection }\end{array}$ & \multicolumn{4}{c}{$\begin{array}{c}\text { Category of PPF based on image pattern and } \\
\text { PBWT-for-height }\end{array}$} \\
\cline { 2 - 5 } & $\begin{array}{c}\text { No PPF } \\
\text { (\%) }\end{array}$ & $\begin{array}{c}\text { Indeterminate } \\
\text { PPF (\%) }\end{array}$ & $\begin{array}{c}\text { Definite } \\
\text { PPF (\%) }\end{array}$ & $\begin{array}{c}\text { Not } \\
\text { graded (\%) }\end{array}$ \\
\hline Positive & $89(54.9)$ & $13(8.0)$ & $18(11.1)$ & $3(1.9)$ \\
Negative & $34(21.0)$ & $3(1.9)$ & $2(1.2)$ & $0(0.0)$ \\
Total & $123(75.9)$ & $16(9.9)$ & $20(12.3)$ & $3(1.9)$ \\
\hline
\end{tabular}



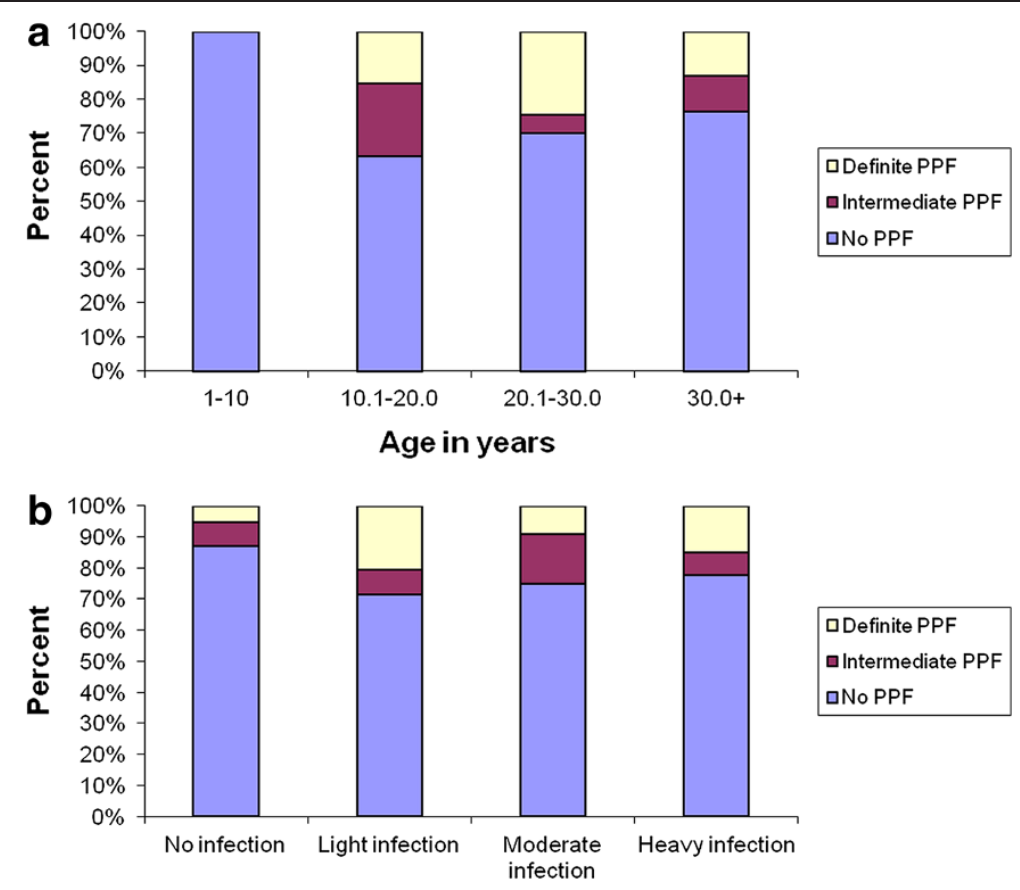

Level of intensity of S. mansoni infection

Figure 4 Distribution of PPF by age (a) and intensity of S. mansoni infection (b), Waja-Timuga, Dec. 2011-Mar. 2012.

supported by some previous studies [16,30]. This could probably be due to similar occupations and water contact patterns to water bodies containing schistosome infected snails. This further also appeared to be attributed to analogous exposure in ecology, socioeconomic status of households, environmental sanitation, and water sources among other factors. All the three mean intensities in the present study sites were categorized under moderate schistosomiasis infection based on WHO [20] classification. The current finding reflects the general pattern of schistosomiasis in that only $17.8 \%$ of those infected are heavily infected but most people are infected moderately and/or lightly, the effects of which are thought to be minimal or ill defined [31].
In recently exposed communities, intensity of schistosomiasis infection increases with child age and then drops again in adulthood [32]. Reports from Jimma town, southwestern Ethiopia showed that intensity of $S$. mansoni infection peaked among individuals in the age ranges $10-19$ years [30] and a similar finding was also reported in Kenya [33], both findings support the current study. These results can indicate that host maturity is an important aspect of resistance to schistosomiasis. Individuals living in areas of endemicity for Schistosoma species who have passed their midteen years generally have significantly less-intense infections than younger children, despite similar exposure to infectious parasites, suggesting that concomitant immunity develops with age in this case [34].

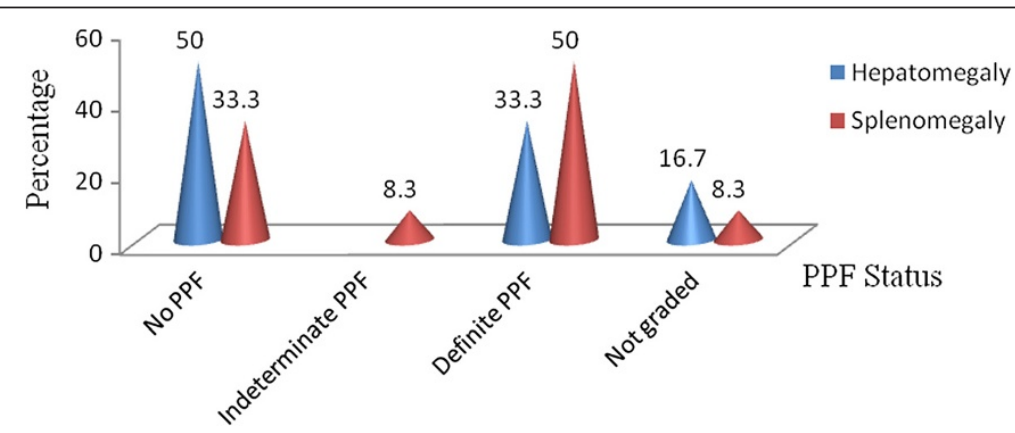

Figure 5 Prevalence of hepatomegaly and splenomegaly stratified by PPF status, Waja-Timuga, Dec. 2011-Mar. 2012. 


$\begin{aligned} & \text { Table } \mathbf{3} \text { Summary of SGOT and SGPT by PPF status } \\
& \text { category, Waja-Timuga, Dec. 2011- Mar. } 2012\end{aligned}$
\begin{tabular}{lccccc}
\hline $\begin{array}{l}\text { PPF } \\
\text { status }^{\text {i }}\end{array}$ & \multicolumn{2}{c}{ No. (\%) SGOT level } & & \multicolumn{2}{c}{ No. (\%) SGPT leveliii } \\
\cline { 2 - 3 } \cline { 5 - 6 } & $\mathbf{0}$ & $\mathbf{1}$ & & $\mathbf{0}$ & $\mathbf{1}$ \\
\hline $\mathbf{0}$ & $3(100)$ & - & & $3(100)$ & - \\
$\mathbf{1}$ & $4(80)$ & $1(20)$ & & $5(100)$ & - \\
$\mathbf{2}$ & $16(94.12)$ & $1(5.88)$ & & $16(94.12)$ & $1(5.88)$
\end{tabular}

i $0=$ no PPF, 1 = indeterminate PPF, $2=$ definite PPF.

ii $0=$ within normal range, $1=$ elevated.

iii $0=$ within range, $1=$ elevated.

Morbidity due to schistosomiasis is caused by a granulomatous response to $S$. mansoni eggs deposited in peripheral portal veins that lead to hepatomegaly, portal hypertension and splenomegaly [35,36]. Previous studies have shown that the degree of morbidity associated with S.mansoni is related to intensity of infection [8,9] and Periportal fibrosis was also linked to infection intensity, as indicated by worm recovery on blood perfusion [37]. In the present study, significant association was observed between schistosomal PPF and intensity of infection. This finding is in line with earlier studies reported by [38,39]. An explanation for these results among individuals could be due to high exposures to potentially infected water bodies, which put the indicated age groups at higher risk of acquiring $S$. mansoni infection.

The proportion of individuals with hepatomegaly decreased and splenomegaly increased with advancing stages of PPF. On the other hand, Berhe et al. [39] reported that prevalence of hepatomegaly and splenomegaly varied significantly by different back ground characteristics. The variability of these results might be due to the similarity in gender-related behavioral and occupational exposures to potentially infected water bodies in the present study area.

Mild to moderate elevations of the liver enzymes are common. Fatty liver, chronic hepatitis $\mathrm{B}$ and $\mathrm{C}$ and long time use of alcohol are among the cause of mild to moderate liver enzyme elevation [40]. In the current study, most individuals who had periportal fibrosis showed liver enzymes within the normal range. This could probably be due to the small number of study individuals employed in the tests. Moreover, the precise levels of these enzymes do not correlate well with the extent of liver damage or the prognosis. Because these enzymes will be found in blood when there is parenchyma damage and/or in late stage of infection when there is decompensate of liver and presence of co-infection, on the other hand, the study individuals were apparently healthy. Thus, the exact levels of SGOT and SGPT levels cannot be used to determine the degree of liver disease or predict the future [40], however, they are crucial as bio markers of liver function test [41].

\section{Conclusion}

The high prevalence of Schistosoma mansoni infection and schistosomal periportal fibrosis observed in the study area calls for a periodic deworming program to reduce transmission, worm burden and morbidity. Mass praziquantel administration should be complemented with improved sanitation and access to clean water, together with appropriate health education and environmental measures, which are required in order to produce a sustainable impact on transmission of the disease.

\section{Additional file}

Additional file 1: Standard images used during ultrasonography

(Berhe et al., 2007).

\section{Abbreviations}

CSA: Central statistical agency of Ethiopia; EPG: Eggs per gram; IU: International unit; L: Liter; Masl: Meter above sea level; PBWT: Portal branch wall thickness; PPF: Periportal fibrosis; SD: Standard deviation; SGOT: Serum glutamic-oxaloacetic transaminase; SGPT: Serum glutamatepyruvate transaminase; WHO: World Health Organization.

\section{Competing interests}

The authors have no competing interests. Hence I, the undersigned, declare that this thesis is my original work and has not been presented for a degree in any other university and that all sources of materials used for the thesis have been duly acknowledged.

Investigator's name: Nigus Abebe Shumuye.

\section{Authors' contributions}

Dr Nega Berhe carried out ultrasonographic examinations, participated in the design and coordination of the study and in proof reading of the manuscript. Prof. Berhanu Erko participated in design and coordination of the study and in proof reading of the manuscript. Dr Girmay Medhin participated in the study design and performed the statistical analysis and in proof reading of the manuscript. All authors read and approved the final version of the manuscript.

\section{Authors' information}

Nigus Abebe (BSc, MSc) College of Veterinary Medicine, Mekelle University. - Lecturer at Mekelle University.

- Interested and working in the area of parasitology and infectious diseases such as parasitic diseases, vector borne diseases, zoonotic diseases.

Nega Berhe (MD, PhD) Aklilu Lemma Institute of Pathobiology, Addis Ababa University.

$\rightarrow$ Clinical researcher at Addis Ababa University.

$\rightarrow$ Working in the areas of Visceral Leishmaniasis, Schistosomiasis mansoni, HIV Infections, Endemic Diseases and AIDS-Related Opportunistic Infections. Berhanu Erko, Professor of Medical Parasitology, Aklilu Lemma Institute of Pathobiology, Addis Ababa University.

$\rightarrow$ Working in areas of parasitology such as Schistosomiasis mansoni, Helminthiasis, Intestinal Parasitic Diseases, Ascariasis and Falciparum Malaria. Girmay Medhin (BSC, MSc, PhD) Bio-Statistician, epidemiologist, Aklilu Lemma Institute of Pathobiology, Addis Ababa University.

$\rightarrow$ Interested and working in the area of Schistosomiasis mansoni, Mental Disorders, Schizophrenia, Helminthiasis and Latent Tuberculosis.

\section{Acknowledgements}

The authors would like to acknowledge Aklilu Lemma Institute of Pathobiology (ALIPB), Addis Ababa University, the research project entitled "the role of antioxidant rich dietary supplementation in the reversal of schistosomal periportal fibrosis" led by Dr Nega Berhe, ALIPB and College of Veterinary Medicine, Mekelle University for provision of material and financial support to carry out the study. My gratitude is also extended to communities of Timuga health center, 
Mr. Berihun Sisay and Mr. Sisay Dessie for their technical assistance. I would also like to thank the community (local people) of Waja-Timuga who participated in the study.

\section{Author details}

${ }^{1}$ Mekelle University, College of Veterinary Medicine, Mekelle, Tigray, Ethiopia. ${ }^{2}$ Addis Ababa University, Aklilu Lemma Institute of Pathobiology, Addis Ababa, Ethiopia.

\section{Received: 1 August 2013 Accepted: 21 March 2014}

Published: 1 April 2014

\section{References}

1. Teklehaimanot A, Fletcher M: Parasitological and malacological survey of schistosomiasis mansoni in the Beles Valley, northwestern Ethiopia. J Trop Med Hyg 1990, 93(1):12-21.

2. Ali A, Erko B, Woldemichael T, Kloos H: Schistosomiasis. In The Epidemiology and Ecology of Health and Diseases in Ethiopia. 1st edition. Edited by Berhane $Y$, Hailemariam D, Kloos H. Addis Ababa: Shama books; 2006:660-673.

3. Vennervald B, Dunne D: Morbidity in Schistosomiasis: an update. Curr Opin Infect Dis 2004, 17(5):439-447.

4. Booth M, Vennervald B, Kabatereine $\mathrm{N}$ : Hepatosplenic morbidity in two neighboring communities in Uganda with high levels of Schistosoma mansoni infection but very different durations of residence. Trans $R$ Soc Trop Med Hyg 2004, 98:125-136.

5. Miguel S: Immunoregulation of schistosomiasis. USA: Tufts University; 2011.

6. Dunne D, Pearce E: Immunology of hepatosplenic schistosomiasis mansoni: a human perspective. Microbes Infect 1999, 1:553-560.

7. Cheever A, Hoffmann K, Wynn T: Immunopathology of schistosomiasis mansoni in mice and men. Immunol Today 2000, 21:465-466.

8. Ross A, Bartley P, Sleigh A, Olds G, Williams G, McManus D: Schistosomiasis. N Engl J Med 2002, 346(16):1212-1220.

9. van der Werf M, de Vlas S, Brooker S, Looman C, Nagelkerke N, Habbema J, Engels D: Quantification of clinical morbidity associated with Schistosome infection in Sub-Saharan Africa. Acta Trop 2003, 86(2):125-139.

10. Stothard R: Improving control of African Schistosomiasis: towards effective use of rapid diagnostic tests within an appropriate disease surveillance model. Trans R Soc Trop Med Hyg 2009, 103(4):325-332.

11. Richter J, Hatz C, Häussinger D: Ultrasound in tropical and parasitic diseases. Lancet 2003, 362(9387):900-902.

12. Kabatereine N, Kemijumbi J, Ouma J, Kariuki H, Richter J, Kadzo H, Madsen $\mathrm{H}$, Butterworth A, Ornbjerg N, Vennervald B: Epidemiology and morbidity of Schistosoma mansoni infection in a fishing community along Lake Albert in Uganda. Trans R Soc Trop Med Hyg 2004, 98(12):711-718.

13. King C, Magak P, Salam A, Ouma J, Kariuki C, Blanton R: Measuring morbidity in Schistosomiasis mansoni: relationship between image pattern, portal vein diameter and portal branch thickness in large-scale surveys using new WHO coding guidelines for ultrasound in schistosomiasis. Trop Med Int Health 2003, 8:109-117.

14. Erko B, Tedla S, Wolde-Yohannes L: Current status of schistosomiasis in Ethiopia. In Aklilu Lemma International Memorial Symposium Proceedings PP.48-59. Faculty of Science. Addis Ababa, Ethiopia: Addis Ababa University; 1997

15. Tadesse G: The prevalence of intestinal helminthic infections and associated risk factors among school children in Babile town, eastern Ethiopia. Ethiop J Health Dev 2005, 19:140-147.

16. Dejenie T, Petros B: Irrigation practices and intestinal helminth infections in Southern and Central zones of Tigray. Ethiop J Health Dev 2009, 23:48-56.

17. CSA: The 2006 Population and Housing Census of Ethiopia. Addis Ababa: Results for the Tigray Region, CSA; 2007

18. Tadesse D, Tsehay A, Mahmud A: Efficacy of praziquantel in treating $S$. mansoni infected school children in Timuga and Waja, North Ethiopia. MEJS 2010, 2(2):5-6.

19. WHO: Basic Laboratory Methods in Medical Parasitology. World Health Organization. Geneva: WHO Technical Report Series; 1991:749.

20. WHO: Prevention and control of schistosomiasis and soil-Transmitted Helminthiasis. WHO Technical Report Series 2002, 912:1-57.

21. WHO: Ultrasound in Schistosomiasis: A Practical Guide to the Standardized Use of Ultrasonography for the Assessment of Schistosomiasis related Morbidity; 2011. http://www.who.int/tdr/publications/publications/pdf/ultrasound.pdf; 2000, Accessed on August 20, 2011
22. Wang CS, Chang T-T, Yao W-J, Wang S-T, Chou P: Impact of increasing alanine aminotransferase levels within normal range on incident diabetes. J Formos Med Assoc 2012, 111(4):201-208.

23. Alemayehu T, Yebiyo Y, Ghebreyesus T, Wtten K: Malaria, Schistosomiasis and Intestinal Helminthes in relation to microdams in Tigray, Northern Ethiopia. Parasitol 1998, 40:259-267.

24. Erko B, Medhin G, Berhe N, Abebe F, Gebre-Michael T, Gundersen SG: Epidemiological studies on intestinal schistosomiasis in Wondo Genet, southern Ethiopia. Ethiop Med J 2002, 40(1):29-39.

25. Amsalu G: Epidemiology of intestinal schistosomiasis in Hayk town, Northeast Ethiopia. In MSc thesis. Addis Ababa University, School of Graduate Studies, Addis Ababa University Printing Press: Addis Ababa University, School of Graduate Studies, Addis Ababa University Printing Press; 2012. URI: http://etd.aau.edu.et/dspace/handle/123456789/2231.

26. Legesse $M$, Erko B: Prevalence of intestinal parasites among schoo children in a rural area close to Lake Langano in the southeast Ethiopia. Ethiop J Health Dev 2004, 18:116-120.

27. Birrie H, Tedla S, Tilahun G, Kloss H, Eshete H: Schistosomiasis and its distribution in Ethiopia and Eritrea. In Schistosomiasis in Ethiopia and Eritrea. Edited by Birrie H, Tedla S, Jemaneh L. Institute of Pathobiology, Addis Ababa University Printing Press; 1998:29-89.

28. Assefa T, Wolde-Michael T, Dejene A: Intestinal parasitism among students in three localities in south Wello, Ethiopia. Ethiop J Health Dev 1998, 12:231-235.

29. Wolde-Michael T, Endeshaw T, Shibre T, Gebre T, Haddis M, Tilahun D, Gebre-Yesus L, Dereje S: Intestinal parasitic infection in Western Abaya with a special reference to Schistosomiasis mansoni. Ethiop J Health Dev 1999, 13(1):21-26.

30. Mengistu M, Shimelis T, Torben W, Terefe A, Kassa T, Hailu A: Human intestinal schistosomiasis in communities living near three rivers of Jimma town, south Western Ethiopia. Ethiop J Health Sci 2011, 21(2):3-7.

31. Assis O, Barreto L, Prado S, Reis G, Parraga M, Blanton E: Schistosoma mansoni infection and nutritional status in school children: a randomized, double-blind trial in northeastern Brazil. Am J Clin Nutr 1998, 68:1247-1253.

32. Woolhouse M, Hagan P: Seeking the ghost of worms. Nature Med 1999 5:1225-1227.

33. Handzel T, Karanaja D, Addiss D: Geographic distribution of schistosomiasis and soil transmitted helminthes in western Kenya. Am J Trop Med Hyg 2003, 69(3):318-323.

34. Butterworth A, Curry A, Dunne D, Fulford A, Kimani G, Kariuki H, Klumpp R, Koech D, Mbugua G, Ouma J: Immunity and morbidity in human schistosomiasis mansoni. Trop Geogr Med 1994, 46:197-208.

35. El-Hawey A, Massoud A, El-Badrawy N, El-Garem A, Khalil A, Metwaly A Hepatic histochemistry and electron microscopy of hamsters infected with S. mansoni and treated with praziquantel therapy. J Egypt Soc Parasitol 1985, 15(1):249-262.

36. de Jesus A, Miranda D, Miranda R, Araújo I, Magalhães A, Bacellar M, Carvalho EM: Morbidity associated with Schistosoma mansoni infection determined by ultrasound in an endemic area of Brazil, Caatinga do Moura. Am J Trop Med Hyg 2000, 63(1-2):1-4.

37. Cheever A, Andrade Z: Pathological lesions associated with Schistosoma mansoni infection in man. Trans R Soc Trop Med Hyg 1967, 61:626-639.

38. Kariuki H, Mbugua G, Magak P, Bailey J, Muchiri E, Thiongo F, King C, Butterworth A, Ouma J, Blanton R: Prevalence and familial aggregation of schistosomal liver morbidity in Kenya: evaluation by new ultrasound criteria. J Infect 2001, 183(6):960-966.

39. Berhe N, Mckew S, Gudersn SG: Intensity of Schistosoma Mansoni, Hepatitis B, Age, and Sex Predict Levels of Hepatic Periportal Thickening/Fibrosis (PPT/F): A Large-Scale Community-Based Study in Ethiopia. Am Soc Trop Med Hyg 2007, 77(6):1079-1086.

40. Siamak T: In Liver blood test. Edited by William C. 2012. URL: http://www. medicinenet.com/liver_blood_tests/article.htm; 2012. Accessed on April 25, 2012.

41. Nalpas B, Vassault A, Charpin S, Lacour B, Berthelot P: Serum mitochondrial aspartate aminotransferase as a marker of chronic alcoholism: diagnostic value and interpretation in a liver unit. Hepatol 1986, 6(4):608-614.

doi:10.1186/1756-3305-7-158

Cite this article as: Abebe et al: Clinico-epidemiological study of Schistosomiasis mansoni in Waja-Timuga, District of Alamata, northern Ethiopia. Parasites \& Vectors 2014 7:158. 